PROCEEDINGS OF THE AMERICAN MATHEMATICAL SOCIETY

Volume 124, Number 6, June 1996

\title{
A RESULT ON DERIVATIONS
}

\author{
TSIU-KWEN LEE AND JER-SHYONG LIN
}

(Communicated by Ken Goodearl)

\begin{abstract}
Let $R$ be a semiprime ring with a derivation $d$ and let $U$ be a Lie ideal of $R, a \in R$. Suppose that $a d(u)^{n}=0$ for all $u \in U$, where $n$ is a fixed positive integer. Then $a d(I)=0$ for $I$ the ideal of $R$ generated by $[U, U]$ and if $R$ is 2-torsion free, then $\operatorname{ad}(U)=0$. Furthermore, $R$ is a subdirect sum of semiprime homomorphic images $R_{1}$ and $R_{2}$ with derivations $d_{1}$ and $d_{2}$, induced canonically by $d$, respectively such that $\bar{a} d_{1}\left(R_{1}\right)=0$ and the image of $U$ in $R_{2}$ is commutative (central if $R$ is 2-torsion free), where $\bar{a}$ denotes the image of $a$ in $R_{1}$. Moreover, if $U=R$, then $\operatorname{ad}(R)=0$. This gives Brešar's theorem without the $(n-1)$ !-torsion free assumption on $R$.
\end{abstract}

In [8] I. N. Herstein proved that if $R$ is a prime ring and $d$ is an inner derivation of $R$ such that $d(x)^{n}=0$ for all $x \in R$ and $n$ a fixed integer, then $d=0$. In [6] A. Giambruno and I. N. Herstein extended this result to arbitrary derivations in semiprime rings. In [2] L. Carini and A. Giambruno proved that if $R$ is a prime ring with a derivation $d$ such that $d(x)^{n(x)}=0$ for all $x \in U$, a Lie ideal of $R$, then $d(U)=0$ when $R$ has no nonzero nil right ideals, char $R \neq 2$ and the same conclusion holds when $n(x)=n$ is fixed and $R$ is a 2-torsion free semiprime ring. Using the ideas in [2] and the methods in [5] C. Lanski [11] removed both the bound on the indices of nilpotence and the characteristic assumptions on $R$.

In [1] M. Brešar gave a generalization of the result due to I. N. Herstein and A. Giambruno [6] in another direction. Explicitly, he proved the theorem: Let $R$ be a semiprime ring with a derivation $d, a \in R$. If $a d(x)^{n}=0$ for all $x \in R$, where $n$ is a fixed integer, then $a d(R)=0$ when $R$ is an $(n-1)$ !-torsion free ring. The present paper is then motivated by Brešar's result and by Lanski's paper [11]. We prove Brešar's result without the assumption of $(n-1)$ !-torsion free on $R$. In fact, we study the Lie ideal case as given in [11] and then obtain Brešar's result as the corollary to our main result. More precisely, we shall prove the following

Main Theorem. Let $R$ be a semiprime ring with a derivation $d$ and let $U$ be a Lie ideal of $R, a \in R$. Suppose that $a d(u)^{n}=0$ for all $u \in U$, where $n$ is a fixed integer. Then ad $(I)=0$ for $I$ the ideal of $R$ generated by $[U, U]$ and if $R$ is 2-torsion free, then $\operatorname{ad}(U)=0$.

Furthermore, $R$ is a subdirect sum of semiprime homomorphic images $R_{1}$ and $R_{2}$ with derivations $d_{1}$ and $d_{2}$, induced canonically by $d$, respectively such that

Received by the editors March 28, 1994 and, in revised form, May 9, 1994 and December 9, 1994.

1991 Mathematics Subject Classification. Primary 16W25.

Key words and phrases. Semiprime rings, derivations, Lie ideals, GPIs, differential identities.

(C)1996 American Mathematical Society 
$\bar{a} d_{1}\left(R_{1}\right)=0$ and the image of $U$ in $R_{2}$ is commutative (central if $R$ is 2-torsion free), where $\bar{a}$ denotes the image of a in $R_{1}$.

Corollary. Let $R$ be a semiprime ring with a derivation $d$ and $a \in R$. If $a d(x)^{n}=0$ for all $x \in R$, where $n$ is a fixed integer, then $\operatorname{ad}(R)=0$.

Throughout this paper let $R$ be a semiprime ring, $U$ a Lie ideal of $R, Z$ the center of $R, C$ the extended centroid of $R$ and $d$ a derivation of $R$. Given two elements $a, b \in R,[a, b]$ will denote the element $a b-b a$; also, for two subsets $A$ and $B$ of $R$, $[A, B]$ is then the additive subgroup of $R$ generated by all elements $[a, b]$ with $a \in A$, $b \in B$. For any subset $S$ of $R$, denote by $r_{R}(S)$ the right annihilator of $S$ in $R$, that is, $r_{R}(S)=\{x \in R \mid S x=0\}$ and $l_{R}(S)$ is defined similarly. If $r_{R}(S)=l_{R}(S)$,

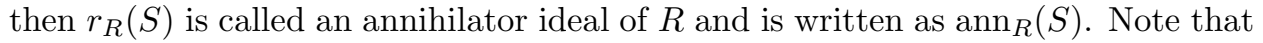
it is easy to check that every annihilator ideal of the semiprime ring $R$ is invariant under all derivations of $R$. This fact will be used in our proofs.

We begin this paper with the following key result.

Theorem 1. Let $R$ be a prime ring with a derivation $d$ and let $U$ be a Lie ideal of $R, a \in R$. Suppose that ad $(u)^{n}=0$ for all $u \in U$, where $n$ is a fixed integer. Then

(i) $\operatorname{ad}(U)=0$ unless char $R=2$ and $\operatorname{dim}_{C} R C=4$.

(ii) $\operatorname{ad}(R)=0$ if $[U, U] \neq 0$.

Proof. We first prove (i). If $U$ is central, then $d(U) \subseteq Z$ since $d(Z) \subseteq Z$. Since every nonzero central element in the prime ring $R$ is not a zero-divisor, we have $\operatorname{ad}(U)=0$ by our assumption. Assume next that $U$ is a noncentral Lie ideal of $R$. We assume further that either char $R \neq 2$ or $\operatorname{dim}_{C} R C>4$. By [10, Theorem 13], $[U, U] \neq 0$ and $0 \neq[I, R] \subseteq U$, where $I$ is the ideal of $R$ generated by $[U, U]$. In this case we want to prove $\operatorname{ad}(R)=0$. Suppose on the contrary that $\operatorname{ad}(R) \neq 0$.

By our assumption we have $a d([x, y])^{n}=0$ for all $x, y \in I$. Thus

$$
a([d(x), y]+[x, d(y)])^{n}=0
$$

for all $x, y \in I$. Denote by $Q$ the two-sided Martindale quotient ring of $R$. If $d$ is not inner on $Q$, applying Kharchenko's theorem [9] we have $a([z, y]+[x, w])^{n}=0$ for all $x, y, z, w$, in $R$. In particular, $a[x, y]^{n}=0$ for all $x, y \in R$. Let $x, y, z, t \in R$ and set $u=[x, y]^{n}$. Then $0=a[z, u t a]^{n}=a(z u t a)^{n}$ since $a u=0$ and hence $(a z u t)^{n+1}=0$. Applying Levitzki's lemma [7, Lemma 1.1] we have $a z u=0$. By the primeness of $R$ it follows that $u=0$ since $a \neq 0$. That is, $[x, y]^{n}=0$ for all $x, y \in R$. Now by [6] or by an easy computation we can conclude that $R$ is commutative, a contradiction. Thus we may always assume that $d$ is $Q$-inner. So there exists an element $b \in Q$ such that $d(x)=[b, x]$ for all $x \in R$. Since by [3] $Q$ and $I$ satisfy the same generalized polynomial identities (or GPIs in brief), we have $a[b,[x, y]]^{n}=0$ for all $x, y \in Q$. Also, since $Q$ remains prime by the primeness of $R$, replacing $R$ by $Q$ we may assume that $b \in R$ and $C$ is just the center of $R$. Note that $R$ is a centrally closed prime $C$-algebra in the present situation [4]. We divide the proof into two cases.

Case 1. Assume that $R$ satisfies a nonzero GPI.

In this case, since $R$ is a centrally closed prime $C$-algebra, by Martindale's theorem [12] $R$ is a strongly primitive ring. Let ${ }_{R} V$ be a faithful irreducible left $R$-module with commuting ring $D=\operatorname{End}\left({ }_{R} V\right)$. By the density theorem, $R$ acts 
densely on $V_{D}$. For any given $v \in V$ we claim that $v$ and $b v$ are $D$-dependent. Assume first that $a v \neq 0$. Suppose on the contrary that $v$ and $b v$ are $D$-independent. By the density of $R$ in $\operatorname{End}\left(V_{D}\right)$ there exist two elements $x$ and $y$ in $R$ such that

$$
x v=0, \quad x b v=b v \quad \text { and } \quad y v=0, \quad y b v=v .
$$

Then $0=a(b[x, y]-[x, y] b)^{n} v=a v$, a contradiction. Thus $v$ and $b v$ are $D$ dependent as claimed. Assume next that $a v=0$. Since $a \neq 0$, we have $a w \neq 0$ for some $w \in V$. Then $a(v+w)=a w \neq 0$. Applying the first situation we have

$$
b w=w \alpha \quad \text { and } \quad b(v+w)=(v+w) \beta
$$

for some $\alpha, \beta \in D$. But $v$ and $w$ are clearly $D$-independent, and so there exist two elements $x, y$ in $R$ such that

$$
x w=0, \quad x v=v+w \quad \text { and } \quad y w=v+w, \quad y v=v .
$$

Then $[x, y] w=v+w$ and $[x, y](v+w)=w$. Hence

$$
0=a(b[x, y]-[x, y] b)^{n} w= \pm a w(\beta-\alpha)^{n},
$$

which implies $\alpha=\beta$ and hence $b v=v \alpha$ as claimed.

From the above we have proved that $b v=v \alpha(v)$ for all $v \in V$, where $\alpha(v) \in D$ depends on $v \in V$. In fact, it is easy to check that $\alpha(v)$ is independent of the choice of $v \in V$. That is, there exists $\delta \in D$ such that $b v=v \delta$ for all $v \in V$. We claim $\delta \in Z(D)$, the center of $D$. Indeed, if $\beta \in D$, then $b(v \beta)=(v \beta) \delta=v(\beta \delta)$ and on the other hand $b(v \beta)=(b v) \beta=(v \delta) \beta=v(\delta \beta)$. Therefore $v(\beta \delta-\delta \beta)=0$. So $\beta \delta=\delta \beta$, which implies $\delta \in Z(D)$. So $b \in C$ and hence $d=0$, a contradiction.

Case 2. Assume that $R$ does not satisfy any nonzero GPI.

Denote by $C\{X, Y, \ldots\}$ the free $C$-algebra with indeterminates $X, Y, \ldots$, and by $Q *_{C} C\{X, Y, \ldots\}$ the free product over $C$ of the $C$-algebra $Q$ and the free $C$-algebra $C\{X, Y, \ldots\}$. Since $a(b[x, y]-[x, y] b)^{n}=0$ for all $x, y \in R$, we see that

$$
a(b[X, Y]-[X, Y] b)^{n}=0 \quad \text { in } Q *_{C} C\{X, Y, \ldots\}
$$

since $R$ has no nonzero GPI. Expanding this we see that

$$
a b[X, Y](b[X, Y]-[X, Y] b)^{n-1}-a[X, Y] b(b[X, Y]-[X, Y] b)^{n-1}=0 .
$$

Suppose for the moment that $a b$ and $a$ are $C$-independent. Then (1) implies

$$
a b[X, Y](b[X, Y]-[X, Y] b)^{n-1}=0=a[X, Y] b(b[X, Y]-[X, Y] b)^{n-1} .
$$

Since $0=a[X, Y]\left(b^{2}[X, Y]-b[X, Y] b\right)(b[X, Y]-[X, Y] b)^{n-2}$, we have $b^{2}=\mu b$ for some $\mu \in C$; otherwise we get $a([X, Y] b)^{n}=0$ and hence either $a=0$ or $b=0$, a contradiction. Note that by $b^{2}=\mu b$ we have

$$
b[b,[X, Y]]=b[X, Y](\mu-b) \quad \text { and } \quad(b-\mu)[b,[X, Y]]=(\mu-b)[X, Y] b .
$$

Thus

$$
0=a[X, Y] b(b[X, Y]-[X, Y] b)^{n-1}=a[X, Y] b[X, Y](b-\mu)[X, Y] b \cdots[X, Y] t,
$$

where its degree in $[X, Y]$ is $n$ and $t=b$ or $(\mu-b)$. Clearly, this implies $b=\mu$, a contradiction.

Next assume that $a b$ and $a$ are $C$-dependent. Thus $a b=\beta a$ for some $\beta \in C$. Then $a(b-\beta)=0$. But $b$ and $b-\beta$ induce the same inner derivation of $R$, replacing $b$ by $b-\beta$ we may assume from the start that $a b=0$. So we have 
$a[X, Y] b(b[X, Y]-[X, Y] b)^{n-1}=0$. As before, the same argument implies $b \in C$, a contradiction. This gives the proof of (i).

For (ii), since $[U, U] \neq 0$, it follows from the proof of (i) that $a d(R)=0$. This completes the proof of the theorem.

We are in a position to prove the Main Theorem.

Proof of the Main Theorem. Let $P$ be a prime ideal of $R$ such that $[U, U] \nsubseteq P$ and set $\bar{R}=R / P$. Assume first that $d(P) \subseteq P$. Then $d$ induces a canonical derivation $\bar{d}$ on $\bar{R}$. By the assumption, $\bar{a} \bar{d}(\bar{u})^{n}=0$ for all $\bar{u} \in \bar{U}$. Note that $\bar{U}$ is a Lie ideal of $\bar{R}$ such that $[\bar{U}, \bar{U}] \neq 0$ since $[U, U] \nsubseteq P$. It follows from Theorem 1 that $\bar{a} \bar{d}(\bar{R})=0$, that is, $\operatorname{ad}(R) \subseteq P$.

Assume next that $d(P) \nsubseteq P$. Then for any $t \in P, u \in U$ we have $0=a d([t, u])^{n}=$ $a([d(t), u]+[t, d(u)])^{n}$ and hence $\bar{a}[\overline{d(t)}, \bar{u}]^{n}=0$ in $\bar{R}$. Applying Theorem 1 again we have $\bar{a}[\overline{d(t)}, \bar{R}]=0$, that is, $a[d(t), R] \subseteq P$. Thus $a[d(P), R] \subseteq P$. In particular, $a[d(P R), R] \subseteq P$ and hence $a[d(P) R, R] \subseteq P$. So $a d(P)[R, R] \subseteq P$ and hence $\operatorname{ad}(P)\left[R^{2}, R\right] \subseteq P$, which implies either $a d(P) \subseteq P$ or $[R, R] \subseteq P$. Since $d(P) \nsubseteq P$ and $[U, U] \nsubseteq P$, this implies $a \in P$. In particular, $\operatorname{ad}(R) \subseteq P$.

Up to now we have proved that for any prime ideal $P$ of $R$ either $a d(R) \subseteq P$ or $[U, U] \subseteq P$.

Recall that $I$ is the ideal of $R$ generated by $[U, U]$. Then $\operatorname{ad}(R) I \subseteq P$ for all prime ideals $P$ of $R$. Thus $a d(R) I=0$ by the semiprimeness of $R$. Set $J=$ $\operatorname{ann}_{R}\left(\operatorname{ann}_{R}(I)\right)$. Clearly, $a d(R) J=0$. Recall that $d(J) \subseteq J$. Thus $\operatorname{ad}(J) \subseteq$ $J \cap \operatorname{ann}_{R}(J)=0$. Since $I \subseteq J$, we have $\operatorname{ad}(I)=0$ as desired.

Assume that $R$ is a semiprime 2 -torsion free ring. Then $\bigcap P=0$, where the intersection is taken over all prime ideals $P$ of $R$ such that $R / P$ is 2-torsion free. Let $P$ be a prime ideal of $R$ such that $R / P$ is 2-torsion free. If $d(P) \subseteq P$, then $d$ induces a canonical derivation on $R / P$ and hence, by Theorem 1 , ad $(U) \subseteq P$ follows. Suppose that $d(P) \nsubseteq P$. Then by the preceding argument either $\operatorname{ad}(R) \subseteq P$ or $[U, U] \subseteq P$. If $a d(R) \subseteq P$, then we are done. Suppose that $[U, U] \subseteq P$. Then $[\bar{U}, \bar{U}]=0$ in $\bar{R}=R / P$. Since $\operatorname{char} \bar{R} \neq 2$, we must have that $\bar{U}$ is central by [10, Theorem 4]. That is, $[U, R] \subseteq P$. Let $u \in U$ and $x \in R$. Then $0=$ $a d([u, x])^{n}=a([u, d(x)]+[d(u), x])^{n}$ and so $\bar{a}[\overline{d(u)}, \bar{x}]^{n}=0$ in $\bar{R}$. By Theorem 1(ii), $\bar{a}[\overline{d(u)}, \bar{R}]=0$. Therefore, $a[d(U), R] \subseteq P$. In particular, $a\left[d(U), R^{2}\right] \subseteq P$ and hence $a R[d(U), R] \subseteq P$. We may assume that $a \notin P$. Therefore, $[d(U), R] \subseteq P$. That is, $\overline{d(U)}$ is central in $\bar{R}$. By the assumption, $\bar{a} \overline{d(u)^{n}}=0$ for all $u \in U$. Since $\overline{d(u)}$ is central, this implies $\bar{a} \overline{d(u)}=0$. So $a d(U) \subseteq P$ as desired. Thus we have proved that $a d(U) \subseteq P$ when $\operatorname{char} R / P \neq 2$. Consequently, $a d(U) \subseteq \bigcap P$, where $P$ runs over all prime ideals of $R$ such that $R / P$ is 2-torsion free. Therefore $a d(U)=0$, since $\bigcap P=0$.

It remains to prove the last statement of the theorem. Denote by $A$ the intersection of all prime ideals $P$ of $R$ with the property that $a d(R) \subseteq P$ and by $B$ the intersection of all prime ideals $P$ with the property that $[U, U] \subseteq P$. We follow the argument of Lanski [11]. Since $A B=0$ and $\operatorname{ann}_{R}\left(\operatorname{ann}_{R}(A)\right) \operatorname{ann}_{R}(A)=0$, we obtain that $\operatorname{ad}(R) \subseteq A \subseteq \operatorname{ann}_{R}\left(\operatorname{ann}_{R}(A)\right)$ and $[U, U] \subseteq B \subseteq \operatorname{ann}_{R}(A)$. Set $R_{1}=R / \operatorname{ann}_{R}\left(\operatorname{ann}_{R}(A)\right)$ and $R_{2}=R / \operatorname{ann}_{R}(A)$. Then $d$ induces derivations $d_{1}$ and $d_{2}$ on $R_{1}$ and $R_{2}$, respectively, $\operatorname{since} \operatorname{ann}_{R}\left(\operatorname{ann}_{R}(A)\right)$ and $\operatorname{ann}_{R}(A)$ are $d$-invariant. Now it is clear that $\bar{a} d_{1}\left(R_{1}\right)=0$ and the image of $U$ in $R_{2}$ is commutative. Finally, if $R$ is 2 -torsion free, then we can construct the subdirect sum $R$ of $R_{1}$ and $R_{2}$ such 
that the image of $U$ in $R_{2}$ is central. Indeed, in this case we can take only these prime ideals $P$ of $R$ with char $R / P \neq 2$ in the construction of $A$ and $B$. By [10, Theorem 4], if $[U, U] \subseteq P$, then $[U, R] \subseteq P$ and hence $[U, R] \subseteq B$. This implies the image of $U$ in $R_{2}$ is central. This finishes the proof of the Main Theorem.

We conclude this paper with proving the Corollary, a generalization of Brešar's result.

Proof of the corollary. By the assumption, $\operatorname{ad}(x)^{n}=0$ for all $x \in R$. By the Main Theorem, $\operatorname{ad}(R)[R, R]=0$. In particular, $\operatorname{ad}(R)\left[R^{2}, R\right]=0$ and hence $\operatorname{ad}(R) R[R, R]=0$. Therefore,

$$
[R, \operatorname{ad}(R)] R[R, \operatorname{ad}(R)]=0,
$$

which implies $a d(R) \subseteq Z$. Let $x \in R$. Then $(a d(x))^{n}=a^{n} d(x)^{n}=a^{n-1}\left(a d(x)^{n}\right)=$ 0 , since $a d(x) a d(x)=a(a d(x)) d(x)=a^{2} d(x)^{2}$. However, since $Z$ is a reduced ring, we have $a d(x)=0$. That is, $\operatorname{ad}(R)=0$ as desired.

\section{REFERENCES}

1. M. Brešar, A note on derivations, Math. J. Okayama Univ. 32 (1990), 83-88. MR 92g:16026

2. L. Carini and A. Giambruno, Lie ideals and nil derivations, Boll. Un. Mat. Ital. 6 (1985), 497-503. MR 87d:16045

3. C. L. Chuang, GPIs having coefficients in Utumi quotient rings, Proc. Amer. Math. Soc. 103 (1988), 723-728. MR 89e:16028

4. J. S. Erickson, W. S. Martindale 3rd, and J. M. Osborn, Prime nonassociative algebras, Pacific J. Math. 60 (1975), 49-63. MR 52:3264

5. B. Felzenszwalb and C. Lanski, On the centralizers of ideals and nil derivations, J. Algebra 83 (1983), 520-530. MR 84m:16028

6. A. Giambruno and I. N. Herstein, Derivations with nilpotent values, Rend. Circ. Mat. Palermo 30 (1981), 199-206. MR 83g:16010

7. I. N. Herstein, Topics in ring theory, Univ. of Chicago Press, Chicago, IL, 1969. MR 42:6018

8. _ Center-like elements in prime rings, J. Algebra 60 (1979), 567-574. MR 80m:16006

9. V. K. Kharchenko, Differential identities of semiprime rings, Algebra and Logic 18 (1979), 58-80. MR 81f: 16052

10. C. Lanski and S. Montgomery, Lie structure of prime rings of characteristic 2, Pacific J. Math. 42 (1972), 117-136. MR 48:2194

11. C. Lanski, Derivations with nilpotent values on Lie ideals, Proc. Amer. Math. Soc. 108 (1990), 31-37. MR 90d:16041

12. W. S. Martindale 3rd, Prime rings satisfying a generalized polynomial identity, J. Algebra 12 (1969), 576-584. MR 39:257

Department of Mathematics, National Taiwan University, Taipei, Taiwan 10764, RePUBLIC OF CHINA

E-mail address: tklee@math.ntu.edu.tw

Department of Mathematics, National Tsing Hua University, Hsinchu, Taiwan 30043, RePublic of China

E-mail address: jslin@math.nthu.edu.tw 